\title{
On the origin of the Fermi arc phenomena in the underdoped cuprates: signature of KT-type superconducting transition
}

\author{
Tao Li and Qiang Han \\ Department of Physics, Renmin University of China, Beijing 100872, P.R.China
}

(Dated: October 31, 2018)

\begin{abstract}
We study the effect of thermal phase fluctuation on the electron spectral function $A(k, \omega)$ in a d-wave superconductor with Monte Carlo simulation. The phase degree of freedom is modeled by a XY-type model with build-in d-wave character. We find a ridge-like structure emerges abruptly on the underlying Fermi surface in $A(k, \omega=0)$ above the KT-transition temperature of the XY model. Such a ridge-like structure, which shares the same characters with the Fermi arc observed in the pseudogap phase of the underdoped cuprates, is found to be caused by the vortex-like phase fluctuation of the XY model.
\end{abstract}

PACS numbers:

The origin of the pseudogap phenomena in the underdoped cuprates is among the most hotly debated issues in the high- $\mathrm{T}_{c}$ physics. Existing theories on the pseudogap falls into two categories: while it is taken by many as the evidence for the existence of preformed Cooper pairs and strong phase fluctuation in the normal state 1 4], some people believed that it should be attributed to a yet another unidentified order [5, [6]. One puzzle about the pseudogap is its momentum and temperature dependence. While early experiments suggests that the pseudogap may inherit the d-wave structure of the superconducting gap, more detailed ARPES measurement show that this is not the case [7 9$]$. Below $T^{*}$, the pseudogap is first seen in a small momentum region around the antinodal point $(\pi, 0)$. The gaped region enlarges with decreasing temperature until the whole Fermi surface(except the nodal points) is gaped below $T_{c}$. The gradual development of the pseudogap on the Fermi surface leaves the system with a finite segment of ungapped Fermi surface which is called Fermi arc in the literature. The length of the Fermi arc increases with temperature and is reported to have a jump at $T_{c}$ : the Fermi arc emerges abruptly at $T_{c}$.

A metal with a unclosed Fermi surface is a strange animal in the zoo of the Landau Fermi liquid theory. The Fermi surface, by its very definition as an equal-energy contour at the chemical potential, is always closed for any well defined quasiparticle dispersion. It is proposed that the Fermi arc is just a half of a closed pocket-like small Fermi surface whose other half is too weak to be detected by ARPES[10]. Such a scenario is widely adopted in the second kind of theories for the pseudogap(related to an unidentified order). In this paper, we try to understand the Fermi arc phenomena in the framework of the phase fluctuation scenario. In this scenario, the Fermi arc is not a genuine Fermi surface in the momentum space across which a finite jump of the occupation number occur, but a phase fluctuation induced pile up of the spectral weight on the Fermi energy in a d-wave paired state.

The effect of the phase fluctuation on the electronic spectral function have been studied by many authors 11 14]. However, most of these studies are limited to the semiclassical treatment of the phase fluctuation. At the semiclassical level, the phase fluctuation is modeled by a uniform supercurrent with a phenomenological distribution of velocity. The Doppler effect of the quasiparticle moving in such a uniform supercurrent background then causes electronic spectral function to shift in both the momentum and energy. More specifically, when the center of mass momentum of the moving Cooper pairs is $2 q$, the electron pairing then happens between momentum $q+k$ and $q-k$, and the excitation energy become $E_{k}-\vec{v}_{k} \cdot \vec{q}$ to first order in $q$. The net effect of the supercurrent on the electron spectral function is to shift it by $q$ in momentum and $\vec{v}_{k} \cdot \vec{q}$ in energy. Here $\vec{v}_{k}$ is velocity of the electron derived from the bare band dispersion relation.

In a recent study 14], the author made the important observation that in a d-wave superconductor the Doppler effect of the fluctuating supercurrent will cause pile up of the spectral weight exactly at the Fermi energy on the underlying Fermi surface, no matter the detailed distribution of the fluctuating supercurrent. The pile up of the spectral weight results in inverse square root singularity at the Fermi energy in the electronic spectral function, resembling a quasiparticle peak. The key point for this to happen is that in a d-wave superconductor, the quasiparticle has linear dispersion around the gap node. For such a system, the effect of the momentum shift and the energy shift caused by the fluctuating supercurrent cancels each other for $q$ in the nodal direction. For $q$ in general direction, the transverse component of $q_{\perp}$ causes no energy shift in leading order and simply move the gap node along the underlying Fermi surface transversely by a distance of $q_{\perp}$.

The semiclassical approach, though intuitively attractive, has limited value to give quantitative estimate of the phase fluctuation effect. To simulate the actual temperature dependence of the phase fluctuation effect, one must go beyond the long wave length limit and take into 
account of the singular effect of the vortex excitation on the quasiparticle motion. Even for the simulation of the phase fluctuation involved in the far field of the vortex, the semiclassical approach has the problem that it is unable to provide a quantitative measure of the strength of the fluctuation.

In this paper, we report the results of the Monte Carlo simulation of the effect of thermal phase fluctuation on the electronic spectral function in d-wave superconductors. The phase fluctuation is described in our work by a XY-type model with a build-in d-wave character. The phase fluctuation then couples to the quasiparticles of the d-wave superconductor via a standard pairing model with the magnitude of the pairing potential $\Delta$ fixed. The simulation shows clearly how the Fermi arc emerge abruptly above the KT transition temperature of the XY model and then increase in strength with temperature. These results indicate that the Fermi arc observed in the underdoped cuprates is a phase fluctuation effect. The abrupt emergence of the Fermi arc above $T_{c}$ suggests that the superconducting transition of the underdoped cuprates are of KT transition-type.

The phase degree of freedom is described by the following XY model

$$
\mathrm{H}_{\text {phase }}=J \sum_{<\alpha, \beta>} \cos \left(\theta_{\alpha}-\theta_{\beta}\right),
$$

in which $\theta_{\alpha}$ is the phase variable defined on the bonds of a square lattice on which the electrons reside. The center of these bonds form another square lattice(see Fig.1) and the sum in Eq.(1) is over neighboring sites of the latter square lattice. The coupling constant $J$ is set to be positive so that a d-wave-like phase structure is favored at low temperature in this model, namely the phase variable on the neighboring bonds tends to have a phase difference of $\pi$. Similar models are also used in other works 12,15$]$.

In principle, the energetics of the phase degree of freedom should be determined by the dynamics of the electron system itself. Here we have treated them as separated degrees of freedom governed by a coupling constant $J$. Except for being more simple to simulate, such a model has the advantage that it takes into account the fact that in the underdoped cuprates the spin pairing and phase coherence are controlled by two separate energy scale $1,[5]$. Such a separation of the energy scales is believed to the key for the formation of the pseudogap phase in most scenarios.

The electron degree of freedom is described by the standard pairing model of the form $\mathrm{H}_{\text {pair }}=\mathrm{H}_{t}+\mathrm{H}_{\Delta}$, in which $\mathrm{H}_{t}$ and $\mathrm{H}_{\Delta}$ are given by

$$
\begin{aligned}
\mathrm{H}_{t}= & -t \sum_{<i, j>, \sigma}\left(c_{i, \sigma}^{\dagger} c_{j, \sigma}+\text { h.c. }\right) \\
& -t^{\prime} \sum_{<<i, j>>, \sigma}\left(c_{i, \sigma}^{\dagger} c_{j, \sigma}+\text { h.c. }\right)-\mu
\end{aligned}
$$

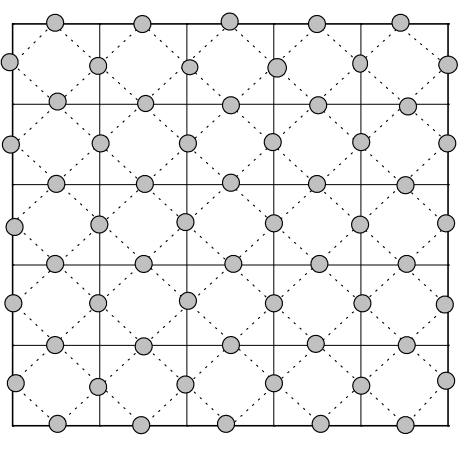

FIG. 1: The lattice on which model Eq.(1) is defined on. The electrons reside on the square lattice made of the solid lines. The phase variables resides on the bonds of this square lattice which are indicated by the gray dots. These gray dots form another square lattice of itself.

$$
\mathrm{H}_{\Delta}=\sum_{<i, j>}\left[\Delta e^{i \theta_{\alpha}}\left(c_{i, \uparrow}^{\dagger} c_{j, \downarrow}^{\dagger}+c_{j, \uparrow}^{\dagger} c_{i, \downarrow}^{\dagger}\right)+h . c .\right]
$$

in which $t$ and $t^{\prime}$ are the hopping integral of the electron on the square lattice between neighboring and next neighboring sites. The next neighboring hopping $t^{\prime}$ is introduced to model the curvature of the real Fermi surface. $\Delta$ denotes the magnitude of the pairing potential and is kept as a constant in the whole simulation. $\theta_{\alpha}$ is the phase angle on the bond between site $i$ and $j$.

In the simulation, we first generate a thermal phase fluctuation configuration from the distribution $\rho=$ $\exp \left(-\mathrm{H}_{\text {phase }} / k_{B} T\right)$. Then we diagonalize the pairing Hamiltonian Eq.(2) with the generated phase fluctuation configuration and calculated electronic spectral function. We then average the electronic spectral function on different phase fluctuation configurations.

For each individual phase fluctuation configuration, the translational invariance of the pairing Hamiltonian is lost. However, when the result is averaged over all possible phase fluctuation configurations the translational invariance is recovered. In the simulation, we have averaged the electron spectral function over all configurations generated from a given one by space translation at each step. After the average translational invariance is recovered and electronic spectral function as a function of momentum $A(k, \omega)$ is given by

$$
A(k, \omega)=\sum_{n}\left|u_{k}^{n}\right|^{2} \delta\left(\omega-E^{n}\right),
$$

in which $u_{k}^{n}=\sum_{i} u_{i}^{n} e^{i k R_{i}} \cdot u_{i}^{n}$ denotes the eigenvectors of the following B-dG equation with eigenvalue $E^{n}$,

$$
\left(\begin{array}{cc}
H_{t, i, j} & H_{\Delta, i, j} \\
H_{\Delta, i, j}^{*} & -H_{t, i, j}
\end{array}\right)\left(\begin{array}{c}
u_{j}^{n} \\
v_{j}^{n}
\end{array}\right)=E^{n}\left(\begin{array}{c}
u_{i}^{n} \\
v_{i}^{n}
\end{array}\right),
$$


in which $H_{t, i, j}=-t \delta_{i, j+R_{\delta}}-t^{\prime} \delta_{i, j+R_{\delta^{\prime}}}-\mu \delta_{i, j}$ and $H_{\Delta, i, j}=\Delta e^{i \theta_{\alpha}} \delta_{i, j+R_{\delta}}$ are the matrix element of $\mathrm{H}_{t}$ and $\mathrm{H}_{\Delta}$. Here sum over repeated indexes is assumed and $R_{\delta}$ and $R_{\delta^{\prime}}$ denotes the neighboring and next neighboring vector on the square lattice. When $A(k, \omega)$ is calculated for a given phase fluctuation configuration, we do the average over the configurations by the standard Monte Carlo procedure

$$
\langle A(k, \omega)\rangle=\frac{\sum e^{-\mathrm{H}_{\text {phase }} / k_{B} T} A(k, \omega)}{\sum e^{-\mathrm{H}_{\text {phase }} / k_{B} T}}
$$

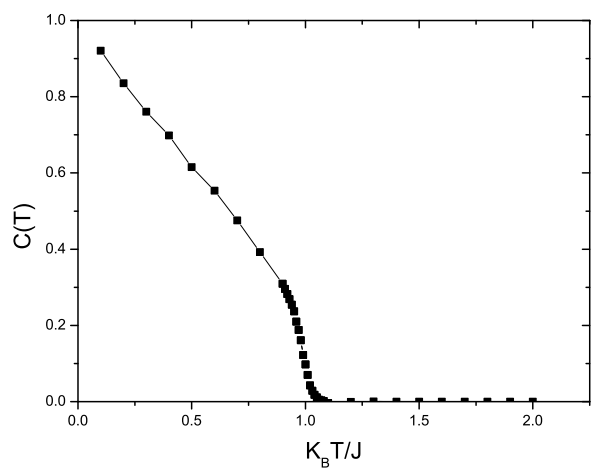

FIG. 2: The correlation(defined in the text) between the phase variables on the most far apart bonds of a $64 \times 64$ lattice as a function of temperature. The abrupt drop of this correlation around $k_{B} T / J \sim 1$ indicates the KT transition of that finite lattice.

The diagonalization of the B-dG Hamiltonian is the most time consuming part of our simulation. At the same time, to resolve the Fermi surface on the finite momentum mesh, we have to study lattice of large enough size. To study relatively larger lattice so that the Fermi surface can be resolved, we adopt the following cutoff strategy. First, we Fourier transform(by FFT algorithm) the BdG Hamiltonian into momentum space. The transformed Hamiltonian reads

$$
\left(\begin{array}{cc}
\xi_{k} \delta_{k, k^{\prime}} & \Delta_{k, k^{\prime}} \\
\Delta_{k, k^{\prime}} & -\xi_{k} \delta_{k, k^{\prime}}
\end{array}\right)\left(\begin{array}{c}
u_{k^{\prime}}^{n} \\
v_{k^{\prime}}^{n}
\end{array}\right)=E^{n}\left(\begin{array}{c}
u_{k}^{n} \\
v_{k}^{n}
\end{array}\right)
$$

in which $\xi_{k}=-2 t\left(\cos k_{x}+\cos k_{y}\right)-4 t^{\prime} \cos k_{x} \cos k_{y}-\mu$, $\Delta_{k, k^{\prime}}=1 / N \sum_{<i, j>} \Delta e^{i \theta_{\alpha}} e^{i k R_{i}-i k^{\prime} R_{j}}$. To simulate the Fermi arc phenomena, we only need to calculate the electron spectral function right on the Fermi energy, $A(k, \omega=0)$. Obviously, those momentums for which $\left|\xi_{k}\right| \gg \Delta$ are not expected to contribute significantly to $A(k, \omega=0)$. For this reason, we set a cutoff energy $E_{c}$ and neglect all momentums for which $\left|\xi_{k}\right|>E_{c}$. We then diagonalize the truncated B-dG Hamiltonian below the cutoff energy and calculate the electron spectral function on the Fermi energy. The convergence of such a cutoff strategy can be easily checked by varying the cutoff energy $E_{c}$. In real simulation, we find $E_{c}=5 \Delta$ is large enough.

We now present the results of our simulation. The simulation is done on a $64 \times 64$ lattice. We have used $t^{\prime} / t=-0.3$ and $\Delta / t=0.1$ to model the real system. The coupling strength of the phase variables, $J$, is set as the unit for temperature. The strength of the phase fluctuation can be read out from the correlation function of the phase variables. In Figure 2, we plot the correlation $C(T)=\left\langle\cos \left(\theta_{\alpha}-\theta_{\alpha^{\prime}}\right)\right\rangle$ between the phase variables on the most far apart bonds of the $64 \times 64$ lattice as a function of temperature. For infinite system, such correlation is zero at finite temperature as required by the Wagner-Mermin theorem. The abrupt drop of this correlation signifies the KT transition of the finite system. For our $64 \times 64$ lattice, we find the abrupt drop of the phase correlation occurs around $k_{B} T / J \sim 1$, slightly above the KT transition temperature for the infinite system $\left(k_{B} T \sim 0.8923 J\right)[16,17]$.

To achieve statistical independence of the samples generated from the Monte Carlo procedure, we have used $10^{5}$ local update of the phase variables to generate every new phase fluctuation configuration. The first $10^{4}$ configurations are discarded for thermalization. The electron spectral function is averaged over 1000 independent phase fluctuation configurations. Figure 3 shows the $A(k, \omega=0)$ calculated at six temperatures. Among the six temperatures, one $\left(k_{B} T=0.5 J\right)$ is below the $\mathrm{KT}$ transition point and the other five are above it $\left(k_{B} T=\right.$ $J, 1.1 J, 1.2 J, 1.3 J, 1.4 J)$.

Below the KT transition temperature $\left(T_{K T}\right)$, the phase fluctuation effect is almost totally quenched and the electron spectral function at the Fermi energy is composed of four sharp peaks at the four nodal points, as in an ideal d-wave superconductor. Right above the KT transition temperature, a ridge-like structure in $A(k, \omega=0)$ emerges on the underlying Fermi surface 18]. The intensity of this ridge-like structure increases rapidly in a small temperature region between $k_{B} T=J$ and $k_{B} T=1.1 J$. For $k_{B} T>1.1 J$, the increase of electron spectral function on the Fermi energy becomes much slower. Such temperature dependence of the electron spectral function at the Fermi energy is in close resemblance to that observed in experiments [8]. It should be noted that the ridge-like structure emerges simultaneously on the whole Fermi surface, though it is clear anisotropic on the Fermi surface. However, considering the finite resolution of the ARPES measurement, it is still reasonable to define a finite segment of the Fermi surface as the Fermi arc for each temperature by setting some threshold for the spectral weight. The arc length defined in such a way clearly increases with temperature.

To quantify the temperature dependence of the electron spectral function in a more objective way, we plot in Figure 4 the integration of the electron spectral function 
(a)
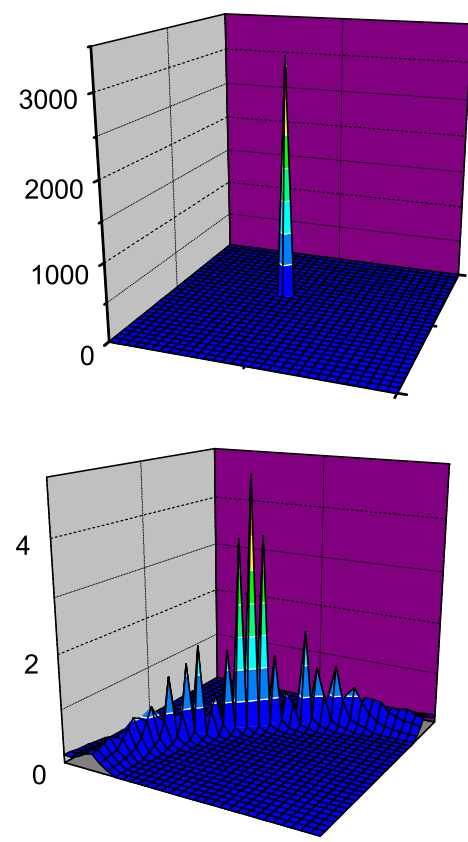

(d) (b)
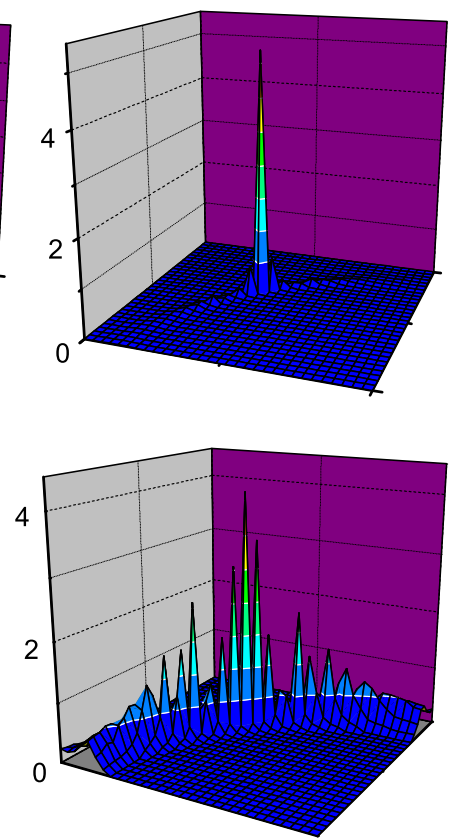

(e) (c)
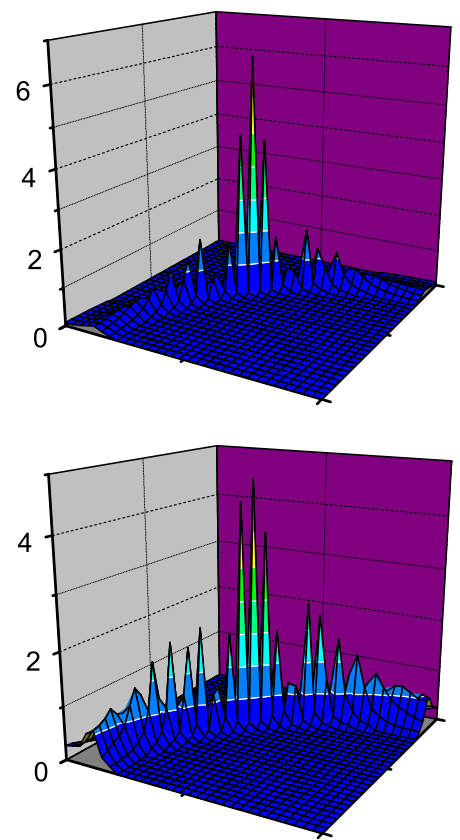

(f)

FIG. 3: The temperature dependence of the electron spectral function at the Fermi energy $A(k, \omega=0)$. (a) $k_{B} T=0.5 J$, (b) $k_{B} T=J,(\mathrm{c}) k_{B} T=1.1 J,(\mathrm{~d}) k_{B} T=1.2 J$, (e) $k_{B} T=1.3 J,(\mathrm{f}) k_{B} T=1.4 J$. Shown here are the results in the first quadrant of the Brillouin zone only.

at Fermi energy in the Brillouin zone $W(\omega=0, T)=$ $\int d k A(k, \omega=0)$ as a function of temperature. The quench of the phase fluctuation effect below $T_{K T}$ and the abrupt emergence of the ridge-like structure above it can be clearly seen in this figure.

We thus conclude that the main features of the experimental observations concerning the Fermi arc phenomena can be accounted for by our phase fluctuation model. The quench of the phase fluctuation effect below $T_{K T}$ and the its abrupt emergence above $T_{K T}$ indicates that Fermi arc phenomena is controlled by the same mechanism that induce the KT transition, namely the vortex excitation. Indeed, according to the study of the XY model, the vortex density enjoys a proliferation just above the KT transition point. With these understanding, it is tempting to argue that the Fermi arc behavior observed in the high-Tc cuprates provide strong evidence that the superconducting transition in the underdoped cuprates are of KT transition-type.

This work is supported by NSFC Grant No. 10774187, National Basic Research Program of China No.2007CB925001 and Beijing Talents Program.

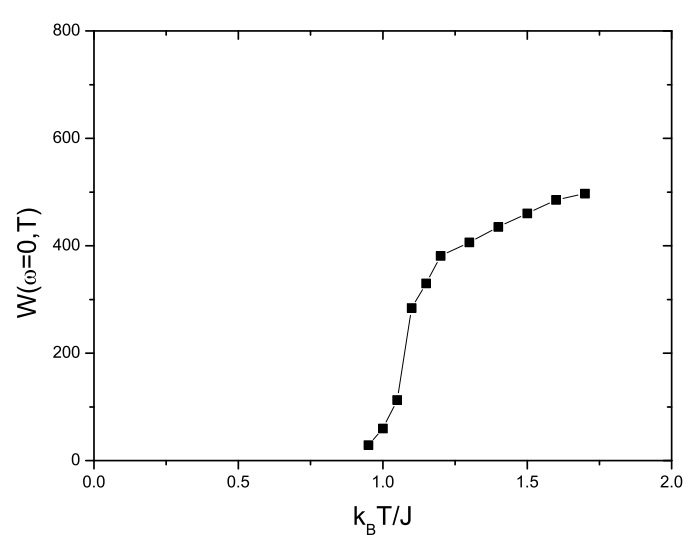

FIG. 4: The integrated electron spectral function over momentum at the Fermi energy $W(\omega=0, T)=\int d k A(k, \omega=0)$ as a function of temperature. 
[1] Y.J. Uemura et al., Phys. Rev. Lett. 62, 2317 (1989).

[2] V.J.Emery and S.A.Klvelson, Nature. Vol 374, 434 (1995).

[3] J. Corson, R. Mallozzi, J. Orenstein, J. N. Eckstein and I. Bozovic, Nature 398, 221 (1999).

[4] Z.A. Xu, N.P. Ong, Y. Wang, T. Kakeshita and S. Uchida, Nature 406, 486 (2000).

[5] P.A. Lee, N. Nagaosa and X-G Wen, Rev. Mod. Phys. 78,17 (2006).

[6] S. Chakravarty, R.B. Laughlin, D.K. Morr, C. Nayak, Phys. Rev. B 63 (2001) 94503.

[7] H. Ding, T. Yokoya, J. C. Campuzano, T. Takahashi, M. Randeria, M. R. Norman, T. Mochiku, K. Kadowaki and J. Giapintzakis, Nature. Vol 382, 51 (1996) .

[8] A.Kanigel et al., Nature Phys. Vol 2, 447 (2006).

[9] A. Damascelli, Z. X. Shen and Z. Hussain, Rev. Mod. Phys. 75, 473 (2003).

[10] N. Doiron-Leyraud, C. Proust, D. LeBoeuf, J. Levallois, J.-B. Bonnemaison, R. Liang, D. A. Bonn, W. N. Hardy, and L. Taillefer, Nature 447, 565 (2007).

[11] M. Franz and A. J. Millis, Phys. Rev. B 58, 14572 (1998).

[12] T. Eckl, D. J. Scalapino, E. Arrigoni, and W. Hanke, Phys. Rev. B 66, 140510(R) (2002).

[13] Matthias Mayr, Gonzalo Alvarez, Cengizen, and Elbio Dagotto , Phys. Rev. Lett. 94, 217001 (2005).

[14] Erez Berg and Ehud Altman, Phys. Rev. Lett. 99, 247001 (2007).

[15] Z. Tesanovic, Nature Physics 4, 408 (2008).

[16] Peter Olsson, Phys. Rev. B 52, 4526 (1995).

[17] Martin Hasenbusch, J. Phys. A: Math. Gen. 38, 5869 (2005).

[18] The strong oscillation of the electron spectral function on the Fermi surface in Fig.3 is not due to the statistical error. The oscillaition has a fixed pattern and is caused by the finite lattice size effect. On a finite lattice, the momentum mesh in the Brillouin zone are not dense enough to resolve all the momentum on the Fermi surface and as a result some Fermi surface momentums are missed(or approximated by nearby momentums). 